\title{
Association of Cartridge Based Nucleic Acid Amplification Test (CBNAAT) Grading on the Basis of Cycle Threshold Value with Conventional Microbiological Diagnosis in Pediatric Tuberculosis
}

\author{
Bineeta Kashyap ${ }^{1}$, Neha Gupta ${ }^{2}$, Pooja Dewan ${ }^{3}$, NP Singh ${ }^{4}$, Ashwani Khanna ${ }^{5}$
}

\begin{abstract}
${ }^{1}$ Associate Professor, Department of Microbiology, University College of Medical Sciences \& Guru Teg Bahadur Hospital, New Delhi, India; ${ }^{2}$ Post graduate student, Department of Microbiology, University College of Medical Sciences \& Guru Teg Bahadur Hospital, New Delhi, India; ${ }^{3}$ Professor, Department of Pediatrics, University College of Medical Sciences \& Guru Teg Bahadur Hospital, New Delhi, India; ${ }^{4}$ Director Professor and Head, Department of Microbiology, University College of Medical Sciences \& Guru Teg Bahadur Hospital, New Delhi, India; ${ }^{5}$ State TB Officer, Revised National TB Control Programme (RNTCP), New Delhi, India
\end{abstract}

[Received: 12 July 2020; Accepted: 30 August 2020; Published: 1 December 2020]

\begin{abstract}
Background: Microbiological confirmation of tuberculosis disease in children remains difficult due to paucibacillary disease and inability to obtain optimal samples. Recently introduced Cartridge based nucleic acid amplification test (CBNAAT) has improved microbiological diagnosis in pediatric tuberculosis. Objectives: We aimed to study association of CBNAAT grading based on cycle threshold value with conventional microbiological diagnosis. Methodology: This prospective study was conducted over a period from November 2016 to October 2017 in the Departments of Microbiology and Pediatrics, University College of Medical Sciences and Guru Teg Bahadur Hospital, Delhi. CBNAAT positive pediatric TB cases $\leq 12$ years were recruited and subjected to Ziehl-Neelsen staining for acid fast bacilli (AFB) \& culture on Lowenstein Jensen medium. CBNAAT positivity was graded based on cycle threshold value: very low, low, medium and high. Results: Smear and culture positivity was highest (100\%) among specimens with high positive CBNAAT result based on CT value. Time to culture positivity was inversely related to CBNAAT grading $(\mathrm{p}=0.000)$. Conclusion: CBNAAT grading has significant positive association with smear and culture positivity. [Bangladesh Journal of Infectious Diseases, December 2020;7(2):72-77]
\end{abstract}

Keywords: CBNAAT; CT value; pediatric; tuberculosis

Correspondence: Dr. Bineeta Kashyap, Flat no. C-402, Vimal CGHS LTD., Plot-3, Sector-12, Dwarka, New Delhi-78, Delhi, India; Email: dr bineetakashyap@yahoo.co.in; Cell no.: +9899583514

Conflict of interest: Nil

Funding agency: Nil

Contribution to authors: BK contributed in the concept, design, definition of intellectual content, clinical studies, experimental studies, data acquisition, manuscript preparation, manuscript editing and manuscript review. NG contributed in the design, definition of intellectual content, literature search, clinical studies, experimental studies, data acquisition, data analysis, statistical analysis and manuscript preparation. PD contributed in design, definition of intellectual content, clinical studies, data acquisition, manuscript editing and manuscript review. NPS contributed in design, definition of intellectual content, experimental studies, data acquisition and manuscript review. AK contributed in the design, experimental studies, data acquisition, manuscript preparation and manuscript review.

How to cite this article: Kashyap B, Gupta N, Dewan P, Singh NP, Khanna A. Association of Cartridge Based Nucleic Acid Amplification Test (CBNAAT) Grading on the Basis of Cycle Threshold Value with Conventional Microbiological Diagnosis in Pediatric Tuberculosis. Bangladesh J Infect Dis 2020;7(2):72-77

Copyright: (02020. Kashyap et al. Published by Bangladesh Journal of Infectious Diseases. This article is published under the Creative Commons CC BY-NC License (https://creativecommons.org/licenses/by-nc/4.0/). This license permits use, distribution and reproduction in any medium, provided the original work is properly cited, and is not used for commercial purposes. 


\section{Introduction}

Despite the discovery of effective and affordable anti tubercular therapy more than fifty years back, TB remains one of the top ten causes of mortality worldwide $^{1}$. Children constitute approximately 10.0 to $20.0 \%$ of the total TB cases in developing economies like India ${ }^{2}$. According to WHO Global Tuberculosis Report 2018 estimated total incidence of TB in India in 2017 was 27.4 lakhs of which 2.24 lakhs cases were contributed by the pediatric age group ${ }^{3}$.

Children may present with vague symptoms mimicking other common childhood diseases. Moreover, microbiological diagnosis also remains difficult in children due to paucibacillary disease and suboptimal specimens leading to diagnostic dilemma $^{2}$. The sensitivity of culture for Mycobacterium tuberculosis, considered as gold standard in adult cases, remains less than 30 to $40 \%$ in pediatric cases ${ }^{4}$. These factors lead to diagnostic delays and hence, pediatric TB remains underreported. There is no gold standard for diagnosis of TB in children ${ }^{4}$. Therefore in most cases, the clinician has to rely on clinical diagnosis based on history, tuberculin skin test, contact tracing, radiology and lack of response to antibiotics.

Cartridge based nucleic acid amplification test (CBNAAT) which was recently introduced and is now recommended by WHO and Revised National Tuberculosis Control Program (RNTCP) as preliminary diagnostic tool among children, has proved to be a breakthrough in the diagnosis of TB in pediatric age group. It provides rapid identification and rifampicin resistance from direct specimen within 2 hours ${ }^{5}$. In this heminested rtPCR assay Mycobacterium tuberculosis is detected by five overlapping molecular probes complementary to the entire $81 \mathrm{bp}$ rрoB core region. Mycobacterium tuberculosis is detected when at least 2 of the five probes give positive signals with a cycle threshold (CT) of $\leq 38$ cycles. A semi quantitative estimate of the concentration of bacilli can be defined by CT range ( $>28=$ very low, 22$28=$ low, 16-22=medium, $<16=$ high $)^{6}$. Rifampicin resistance is reported when difference between the first (early CT) and the last (late CT) $M$. tuberculosis specific beacon was $>3.5$ cycles and was reported sensitive if $\leq 3.5$ cycles. ${ }^{6}$ Smear microscopy is usually the first test available for suspected cases of TB. Cultures take a long time to show growth ${ }^{7}$. The use of CBNAAT has reduced the median time to start treatment for AFB smear negative TB from 56 days to 5 days $^{8}$. Few studies have been done to compare conventional microbiological techniques with $\mathrm{CBNAAT}^{7,9-10}$. But similar studies in pediatric age group are lacking. We aimed to study association of CBNAAT grading based on cycle threshold value with conventional microbiological diagnosis in pediatric TB cases.

\section{Methodology}

This prospective study was conducted over a period from November 2016 to October 2017 in the Departments of Microbiology and Pediatrics, University College of Medical Sciences and Guru Teg Bahadur Hospital, Delhi, India after approval by the institutional research ethics committee. Written consent and assent were taken wherever applicable. All clinically suspected pediatric TB cases $\leq 12$ years of age were recruited in the study based on presence of clinical features, suggestive radiography findings and/or history of exposure to an infectious case of TB and/or reactive tuberculin skin test (TST). ${ }^{11}$ Pulmonary TB cases: Either clinically diagnosed or microbiologically confirmed cases with involvement of lung parenchyma, tracheobronchial tree, miliary TB and cases with both pulmonary as well as extrapulmonary features. ${ }^{12}$ Patients presented with features like fever and cough for $\geq 2$ weeks, unexplained significant weight loss, loss of appetite, history of contact with infectious case, suggestive chest radiography and reactive TST. ${ }^{11}$ Extrapulmonary TB cases: Either clinically diagnosed or microbiologically confirmed cases with involvement of organs other than lungs. ${ }^{12}$ Patients presented with features like fever, loss of weight, anorexia and specific symptoms as per the site of involvement with/without supportive radiographic evidence and/ or reactive TST. ${ }^{11}$ Immunocompromised patients and previous history of TB were excluded from this study. Relevant samples from suspected pediatric TB cases were collected in sterile, leak-proof, disposable and appropriately labeled containers in 2 aliquots. One aliquot was subjected to CBNAAT at DOTS centre of the hospital as per the standard protocol. ${ }^{12} \mathrm{~A}$ semiquantitative grading of CBNAAT positive specimen indicating bacillary load was reported on the basis of cycle threshold (CT) value as very low (CT>28), low (CT 22-28), medium (CT 16-22) and high $(C T<16)$. The other aliqout was subjected to Ziehl-Neelsen staining for acid fast bacilli (AFB) and culture on Lowenstein Jensen (LJ) medium. All specimens were handled and processed in biosafety cabinet type II B2. ${ }^{13}$ Non sterile specimens were initially processed using $\mathrm{N}$-acetyl L-cysteine (NALC) - 4\% Sodium Hydroxide $(\mathrm{NaOH})$ method 
and then inoculated onto LJ slants. Cultures were incubated at $37^{\circ} \mathrm{C}$ and were observed weekly for up to 8 weeks before reporting as negative. Cultures were identified by characteristic rough, tough and buff colored colonies and isolates were further confirmed as Mycobacterium tuberculosis complex by MPT64 antigen detection test and no growth on media containing Para nitro benzoic acid ${ }^{14}$. First 50 CBNAAT positive specimens were included in the study. Data was analyzed using SPSS 20.0 software. Non-parametric tests were applied to report significance.

\section{Results}

The age of the patients in this study ranged from 2 months to 12 years (mean $=7.34 \pm 3.93$ years). Of total 50 cases, 27(54\%) were females and 23 were males. Pulmonary specimens constituted a majority of $35 / 50(70 \%)$ while only $15(30 \%)$ were extrapulmonary. AFB smear were positive for 17 (34\%) while culture showed growth of Mycobacterium tuberculosis for $30 \quad(60 \%)$ specimens.

Table 1: Conventional Microbiological Diagnosis of Various Specimens in Pediatric TB

\begin{tabular}{|c|c|c|c|}
\hline \multicolumn{2}{|l|}{ Specimen } & $\begin{array}{c}\text { Positive by Smear } \\
\text { Microscopy, } n=17(\%)\end{array}$ & $\begin{array}{c}\text { Positive by Culture for } M \text {. } \\
\text { tuberculosis, } \mathrm{n}=\mathbf{3 0}(\%)\end{array}$ \\
\hline \multicolumn{2}{|c|}{ Pulmonary $(\mathrm{n}=35)$} & $15 / 35(43 \%)$ & $24 / 35(69 \%)$ \\
\hline \multicolumn{2}{|c|}{ Extrapulmonary $(\mathrm{n}=15)$} & $2 / 15(13 \%)$ & $6 / 15(40 \%)$ \\
\hline \multirow{6}{*}{$\begin{array}{l}\text { Types of } \\
\text { extra- } \\
\text { pulmonary } \\
\text { specimens }\end{array}$} & $\mathrm{CSF}(\mathrm{n}=8)$ & 0 & $2(25 \%)$ \\
\hline & Pus $(n=3)$ & $1(33 \%)$ & $3(100 \%)$ \\
\hline & Pleural fluid $(\mathrm{n}=1)$ & 0 & $1(100 \%)$ \\
\hline & Empyema pus $(\mathrm{n}=1)$ & 0 & 0 \\
\hline & Wound aspirate $(\mathrm{n}=1)$ & $1(100 \%)$ & 0 \\
\hline & Liver aspirate $(\mathrm{n}=1)$ & 0 & 0 \\
\hline \multicolumn{2}{|c|}{ Total $(\mathbf{n}=\mathbf{5 0})$} & $17(34 \%)$ & $30(60 \%)$ \\
\hline
\end{tabular}

The conventional microbiological diagnosis of various specimens in pediatric TB was recorded. Smear positivity and culture positivity was much higher among pulmonary specimens in $43.0 \%$ and $69 \%$ respectively as compared to extrapulmonary in $13.0 \%$ and $40.0 \%$ respectively (Table 1 ).

Maximum number of samples was reported to be "low" positive ( $48 \%$ ) by CBNAAT while minimum number was reported to be "high" positive (12\%). Only pulmonary specimens that are sputum and gastric aspirate were reported as "high" or "medium" positive. All extrapulmonary specimens were very low or low positive except 1 cerebrospinal fluid (CSF) specimen which was reported to be medium positive (Table 2).

Figure I shows status of AFB smear and result of culture on Lowenstein Jensen media in relation to CBNAAT grading. $100 \%$ of the specimens reported as "very low" positive were smear negative while $100 \%$ of those reported as "high" positive were smear positive. Among those reported to be "low" positive, the smear was positive in $21 \%$ of the cases while among cases reported as "medium" positive, $60 \%$ were smear positive.
Again, 100\% of the specimens reported as "very low" positive did not show growth on culture while $100 \%$ of those reported as "high" or "medium" positive showed growth of Mycobacterium tuberculosis in culture. Among specimens reported as "low" positive, $58.0 \%$ specimens showed growth on culture. All specimens in "very low" category were smear and culture negative while all in "high" category were positive for AFB on smear and showed growth of Mycobacterium tuberculosis in culture.

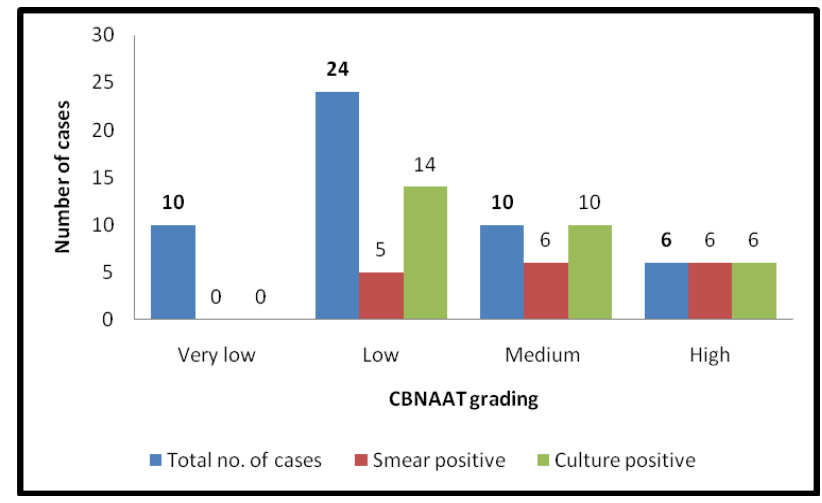

Figure I: AFB Smear Status and Culture Result in Relation to CBNAAT grading 
Table 2: Distribution of Specimens According to CBNAAT Results

\begin{tabular}{|c|c|c|c|c|c|c|}
\hline \multirow[t]{2}{*}{ Specimens } & \multicolumn{4}{|c|}{$\begin{array}{l}\text { CBNAAT Positive Results on The Basis of } \\
\text { Cycle Threshold Value }\end{array}$} & \multirow[t]{2}{*}{ Total } & \multirow[t]{2}{*}{ P value } \\
\hline & Very Low & Low & Medium & High & & \\
\hline GA & 5 & 13 & 6 & 4 & 28 & \multirow{9}{*}{$>0.05$} \\
\hline $\mathrm{CSF}$ & 3 & 4 & 1 & 0 & 8 & \\
\hline Pleural fluid & 0 & 1 & 0 & 0 & 1 & \\
\hline Pus & 0 & 3 & 0 & 0 & 3 & \\
\hline Sputum & 1 & 1 & 3 & 2 & 7 & \\
\hline Empyema pus & 1 & 0 & 0 & 0 & 1 & \\
\hline Liver aspirate & 0 & 1 & 0 & 0 & 1 & \\
\hline Wound biopsy & 0 & 1 & 0 & 0 & 1 & \\
\hline Total & $10(20 \%)$ & $24(48 \%)$ & $10(20 \%)$ & $6(12 \%)$ & 50 & \\
\hline
\end{tabular}

Table 3 shows distribution of specimens reported as "medium" positive and "low" positive on CBNAAT along with their smear and culture status. Pulmonary specimens (GA \& sputum) showed maximum smear positivity and $100.0 \%$ culture positivity among medium positive specimens while among low positive specimens, 5/24 (21.0\%) specimens were smear positive and $14(58.0 \%)$ showed growth of Mycobacterium tuberculosis on culture.

Figure II shows time to culture positivity of the specimens and also correlation of specimens according to CBNAAT grading with time to positivity of cultures. Of 30 positive cultures, maximum number of specimens (40\%) showed growth during $4^{\text {th }}-5^{\text {th }}$ weeks of incubation while $30 \%$ showed growth from $2^{\text {nd }}$ to $3^{\text {rd }}$ weeks and the remaining $30.0 \%$ showed growth from $6^{\text {th }}$ to $8^{\text {th }}$ weeks of incubation. Specimens reported to be "high" positive by CBNAAT showed growth early and latest by $5^{\text {th }}$ week while "medium" positive specimens showed growth over a wide range of incubation period from $2^{\text {nd }}$ week to 8 weeks. No specimen reported as "low" positive showed growth before $4^{\text {th }}$ week. Hence, time to culture positivity was found to be inversely related to CBNAAT grading (spearman's rho $=-0.644$ ) and the correlation was statistically significant $(\mathrm{p}=0.000)$.

Table 3: Smear and Culture Status of Specimens with Medium and Low Positive Result on CBNAAT

\begin{tabular}{|l|c|c|c|c|c|c|}
\hline & \multicolumn{3}{|c|}{ Medium Positive Specimens } & \multicolumn{3}{c|}{ Low Positive Specimens } \\
\hline Sample & Total & $\begin{array}{c}\text { Smear } \\
\text { Positive }\end{array}$ & $\begin{array}{c}\text { Culture } \\
\text { Positive }\end{array}$ & Total & $\begin{array}{c}\text { Smear } \\
\text { Positive }\end{array}$ & $\begin{array}{c}\text { Culture } \\
\text { Positive }\end{array}$ \\
\hline GA & 6 & $3(50 \%)$ & $6(100 \%)$ & 13 & $3(23 \%)$ & $8(61.5 \%)$ \\
\hline Sputum & 3 & $3(100 \%)$ & $3(100 \%)$ & 1 & 0 & $1(100 \%)$ \\
\hline CSF & 1 & 0 & $1(100 \%)$ & 4 & 0 & $1(25 \%)$ \\
\hline Pus & 0 & 0 & 0 & 3 & $1(33.33 \%)$ & $3(100 \%)$ \\
\hline Pleural fluid & 0 & 0 & 0 & 1 & 0 & $1(100 \%)$ \\
\hline Wound aspirate & 0 & 0 & 0 & 1 & $1(100 \%)$ & 0 \\
\hline Liver aspirate & 0 & 0 & 0 & 1 & 0 & 0 \\
\hline Total & $\mathbf{1 0}$ & $\mathbf{6 ( 6 0 \% )}$ & $\mathbf{1 0}(\mathbf{1 0 0 \%})$ & $\mathbf{2 4}$ & $\mathbf{5 ( 2 1 \% )}$ & $\mathbf{1 4}(\mathbf{5 8 \% )}$ \\
\hline
\end{tabular}

Chi-square test was applied. CBNAAT grading was found to have a significant positive association with smear $(\mathrm{p}=0.000)$ and culture $(\mathrm{p}=0.000)$ positivity.

\section{Discussion}

Pediatric TB remains difficult to confirm microbiologically by conventional techniques. WHO recommended upfront CBNAAT for diagnosing $\mathrm{TB}$ in presumptive pulmonary and extra-pulmonary pediatric TB cases ${ }^{15}$. This assay may prove to be a reliable solution to achieve the objective of early and accurate diagnosis of TB and rifampicin resistance which is crucial in pediatric population for early initiation of accurate treatment ${ }^{16}$. 


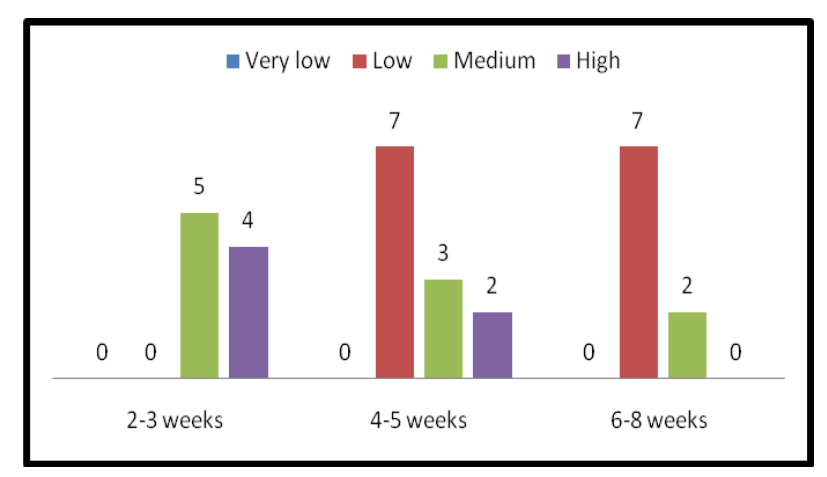

Figure II: Correlation of CBNAAT Grading and Time To Positivity

Since, there is a lack of studies on pediatric population comparing CT values of CBNAAT and conventional microbiological techniques, most of the studies referred to were conducted on adult population. Smear and culture positivity were much higher among pulmonary specimens $(43.0 \%$ and $69.0 \%$ respectively) as compared to extrapulmonary (13\% and $40 \%$ respectively) specimens in this study. $68.0 \%$ of the specimens in this study tested "very low" or "low" positive by CBNAAT. Above findings clearly indicate that in most of the cases, the bacillary load in children remains low which leads to difficulty in diagnosis by conventional methods. Microbiological diagnosis is even more difficult to be established in extrapulmonary TB due to paucibacillary nature of specimens.

In this study, $100 \%$ of the specimens with CT value more than 28 (very low positive) were smear negative; $21.0 \%$ with CT 22 to 28 and $60.0 \%$ with CT 16 to 22 were smear positive while $100.0 \%$ of those with CT value less than 16 (high positive) were positive for AFB on microscopy. Chi-square test was applied which showed that CBNAAT grading had a significant positive association with smear positivity that is higher the grading, higher is the chance of smear being positive. A recent study conducted in Uganda also found a decreasing trend in CT values with increasing grades of smear ${ }^{10}$. About $75.0 \%$ specimens with CT value less than 22 were detected to have AFB on direct microscopy. Similar results were obtained in a recent study on patients with pulmonary $\mathrm{TB}$, where $81.0 \%$ of the cases with cut off value of $\mathrm{CT}$ as 21.1 were identified as smear positive ${ }^{7}$. Hence, low $C T$ value can identify even smear negative specimens with high bacillary load. Since smear negative cases are a possible source of transmission, grading or quantification in CBNAAT could be utilized for identifying such potentially infectious cases ${ }^{7}$. Other studies have also reported strong association of CBNAAT CT values and smear positivity with cut off values ranging from 27.7 to 31.8 with variable sensitivities and specificities ${ }^{17-19}$.

About $100.0 \%$ specimens in this study with CT value up to 22 that is "medium" and "high" positive showed growth of Mycobacterium tuberculosis on LJ media while $100 \%$ of the specimens with CT value $>28$ "very low" positive were culture negative. Among specimens with CT value 22-28 ("low" positive), 58\% showed growth on culture. Hence, higher grade of CBNAAT is associated with significantly increased chances of culture being positive. Such positive correlation between growth on culture and CT value has been seen in previous studies $\operatorname{too}^{9-10}$. In one of those, there was also a decreasing pattern of median CT values associated with increasing categories of culture grade from scanty to +3 on $\mathrm{LJ}$ media ${ }^{10}$.

Time to culture positivity had a significant inverse relation with CBNAAT grading in our study which is also supported by another recent Indian study ${ }^{9}$. Other studies have also suggested strong correlation of time to culture positivity and CT values; and that CT values or CBNAAT grading may be used as a surrogate marker for mycobacterial load and response to treatment in both pulmonary and extrapulmonary $\mathrm{TB}^{17,19-20}$.

\section{Conclusion}

CBNAAT grading has significant positive association with smear and culture positivity. Paucibacillary disease in children is indicated by high percentage of low or very low CBNAAT grading. High and medium CBNAAT grading may be more reliable for initiation of ATT. Wider availability and easy accessibility of CBNAAT might be helpful in diagnosis of at least some cases negative by conventional microbiological techniques. Since culture positivity and time to positivity can act as an indirect measure of bacillary load, cases with "high" and "medium" positive specimens have higher culture positivity rates. These patients need to be put on treatment immediately.

For cases with "very low" positive samples, physician might wait for the culture reports before intervening unless there is a very high suspicion of TB. A strong clinical correlation is advisable to aid decision making in "low" positive cases. Newer diagnostic techniques are needed to be developed which can detect even a very low bacillary load in children. 


\section{References}

1. Glaziou P, Sismanidis C, Floyd K, Raviglione M. Global epidemiology of tuberculosis. Cold Spring Harb Perspect Med. 2015;5:a017798

2. Gupta N, Kashyap B, Dewan P, Hyanki P, Singh NP. Clinical spectrum of pediatric tuberculosis: a microbiological correlation from a tertiary care center. J Trop Pediatr. 2018. https://doi.org/10.1093/tropej/fmy026.

3. World Health Organization. Global tuberculosis report 2018. Geneva: World Health Organization; 2018. Available from:

https://www.who.int/tb/publications/global_report/gtbr2018_an nex2.pdf?ua=1.

4. Kumar MK, Kumar P, Singh A. Recent advances in the diagnosis and treatment of childhood tuberculosis. J Nat Sci Biol Med. 2015;6:314-20

5. Singh S, Singh A, Prajapati S, et al. Xpert MTB/RIF assay can be used on archived gastric aspirate and induced sputum samples for sensitive diagnosis of paediatric tuberculosis. BMC Microbiol. 2015;15:191

6. Lawn SD, Nicol MP. Xpert® MTB/RIF assay: development, evaluation and implementation of a new rapid molecular diagnostic for tuberculosis and rifampicin resistance. Future Microbiol. 2011;6:1067-82

7. Fradejasa I, Ontañónb B, Muñoz-Gallegoa I, RamírezVelaa M.J., López-Roa P. The value of xpert MTB/RIFgenerated CT values for predicting the smear status of patients with pulmonary tuberculosis. J Clin Tuberc Other Mycobact Dis. 2018; 13: 9-12.

8. Nurwidya F, Handayani D, Burhan E, Yunus E. Molecular Diagnosis of Tuberculosis. Chonnam Med J. 2018; 54: 1-9

9. Prakash AK, Datta B, Tripathy JP, Kumar N, Chatterjee $\mathrm{P}$, Jaiswal A.The clinical utility of cycle of threshold value of GeneXpert MTB/RIF (CBNAAT) and its diagnostic accuracy in pulmonary and extra-pulmonary samples at a tertiary care center in India. Indian J Tuberc. 2018;65:296-302.

10. Najjingo I, Muttamba W, Kirenga BJ, Nalunjogi J, Bakesiima R, Olweny F, et al. Comparison of GeneXpert cycle threshold values with smear microscopy and culture as a measure of mycobacterial burden in five regional referral hospitals of Uganda- A cross-sectional study. PLoS ONE. 2019; 14: e0216901

11. Singh V, Mahto D. Antitubercular drugs and RNTCP guidelines for childhood tuberculosis. In: Gupta P, Menon PSN, Ramji S, Lodha R (eds). PG Textbook of Pediatrics: Infections and Systemic Disorders. 2nd ed. New Delhi: Jaypee Brothers Medical Publishers, 2018. pp1348.
12. Central TB Division, Government of India. Technical and operational guidelines for TB control in India 2016. New Delhi: Central TB Division, Directorate General of Health Services, Ministry of Health and Family Welfare, 2016.

13. Forbes BA, Sahm DF, Weissfeld AS. Mycobacteria and other bacteria with unusual growth requirements. In: Forbes BA, Sahn DF, Weissfeld AS (eds). Bailey \& Scott's Diagnostic Microbiology. 13th edn. Missouri: Mosby Elsevier Inc, 2014. pp 484-512.

14. Central TB Division, Government of India. Revised National TB Control Programme Training Manual for Mycobacterium tuberculosis culture and drug susceptibility testing. New Delhi: Central TB Division, Directorate General of Health Services, Ministry of Health and Family Welfare, 2009. http://www.tbcindia.nic.in/showfile.php? lid1/42991. Accessed 3 Oct 2017.

15. World Health Organization. Policy update: automated real time nucleic acid amplification technology for rapid and simultaneous detection of tuberculosis and rifampicin resistance: Xpert MTB/ RIF system for the diagnosis of pulmonary and extrapulmonary TB in adults and children 2013. Geneva: World Health Organization; 2013. Available from: http://apps.who.int/iris/bitstream/10665/112472/1/9789241506 335_eng.pdf

16. Raizada N, Khaparde SD, Salhotra VS, Rao R, Kalra A, Swaminathan S, et al. Accelerating access to quality TB care for pediatric TB cases through better diagnostic strategy in four major cities of India. PLOS ONE. 2018;13: e0193194.

17. Theron G, Pinto L, Peter J, Mishra HK, Mishra HK, van Zyl-Smit R, et al. The use of an automated quantitative polymerase chain reaction (Xpert MTB/RIF) to predict the sputum smear status of tuberculosis patients. Clin Infect Dis. 2012;54:384-88.

18. Lange B, Khan P, Kalmambetova G, Al-Darraji HA, Alland $D$, Antonenka $U$ et al. Diagnostic accuracy of the Xpert ${ }^{\circledR}$ MTB/RIF cycle threshold level to predict smear positivity: a meta-analysis. Int $\mathrm{J}$ Tuberc Lung Dis. 2017;21:493-502.

19. Beynon F, Theron G, Respeito D, Mambuque E, Saavedra B, Bulo H, et al. Correlation of Xpert MTB/RIF with measures to assess Mycobacterium tuberculosis bacillary burden in high HIV burden areas of Southern Africa. Sci Rep. 2018; 8:5201

20. Theron G, Peter J, Calligaro G, Meldau R, Hanrahan $\mathrm{C}$, Khalfey H, et al. Determinants of PCR performance (Xpert MTB/RIF), including bacterial load and inhibition, for TB diagnosis using specimens from different body compartments. Sci Rep. 2014;4:5658 